Originales

\title{
Fiebre secundaria a citostáticos
}

M. J. Oruezábal Moreno*, M. R. Burón Fernández**

\section{Resumen}

- Propósito: la fiebre es uno de los signos clínicos más frecuentes de la práctica médica diaria, siendo el origen farmacológico una de las causas con más baja incidencia.

- Material y métodos: se analizan las manifestaciones clínicas de 85 casos de fiebre diagnosticados entre los años 2001 y 2002 en el Servicio de Oncología Médica de nuestro centro, y se distribuyeron en cinco grupos de acuerdo a su etiología.

- Resultados: se observó que hubo 39 pacientes con fiebre neutropénica, 30 pacientes con fiebre infecciosa no neutropénica, 5 enfermos tuvieron fiebre tumoral y 11 casos, fiebre por fármacos, 6 de aparición precoz y otros 5 tardía.

- Conclusiones: la identificación de las reacciones alérgicas a citostáticos evitaría la administración de antibióticos y la realización de pruebas diagnósticas innecesarias, además de conocer el mecanismo implicado en la alergia a fármacos.

Palabra clave:

Fiebre. Hipersensibilidad. Fiebre medicamentosa.

Oncología, 2004; 27 (3):125-130

* Sección Oncología Médica

** Servicio Medicina Interna

Hospital La Mancha Centro. Alcázar de San Juan (Ciudad Real) 


\section{Summary}

- Purpose: Fever is a very common clinical sign in regular clinical practice. Drugs are responsible for only a small part of the incidence.

- Material and methods: The clinical features of 85 cases of fever diagnosed in 2001 and 2002 in the Medical Oncology Department of our hospital were evaluated and classified into five groups, according to their etiology.

- Results: The findings were the following: 39 patients presented neutropenic fever, 30 cases had fever due to a non-neutropenic infections, in 5 patients fever was of tumor origin, and in 11 cases fever was caused by drugs, 6 of them of early appearance, and the other 5 of late appearance.

- Conclusions: A comprehensive understanding of the hypersensitivity events promoted by cytostatic drugs would avoid needless administration of antibiotics and doing unnecessary tests. It can also help to know the pathogenesis of drug allergy.

Key words: Fever. Allergy. Drugs fever.

\section{Introducción}

Los efectos secundarios de los fármacos son uno de los mayores problemas de la terapia medicamentosa. La mayor parte de ellos se relaciona con la actividad farmacológica del fármaco, pero alrededor del $15 \%$ de estos efectos secundarios se piensa que están mediados de forma inmunológica'.

Clásicamente se consideraba que las alergias a medicamentos estaban mediadas por lgE, pero recientemente se ha postulado un nuevo modelo de reconocimiento de fármacos por células $T$ ya activadas. Este modelo postula la unión directa no covalente del fármaco al complejo MHC-péptido y al TCR, así como a otros receptores farmacológicos lo que determina la estimulación de las células $\mathrm{T}^{2}$.

El reconocimiento de ambos mecanismos fisiopatológicos nos ayudará a comprender la heterogénea variedad de manifestaciones clínicas, permitiendo incluir en el diagnóstico de alergia a fármacos síntomas y signos hasta ahora considerados como reacciones idiosincrásicas por intolerancia fármaco-toxicológica.

La fiebre producida por hipersensibilidad frente a un medicamento ha sido uno de los signos incluidos en los criterios clínicos de alergia a fármacos ${ }^{3}$. Habitualmente, la fiebre por fármacos ocurre en las primeras horas tras la administración y desaparece rápidamente. En muchos casos responde a un mecanismo lgE, aunque a veces la droga induce directamente la degranulación del mastocito con la subsiguiente liberación de mediadores. En otras ocasiones, sin embargo, la fiebre ocurre a partir de las 48 horas tras la administración del fármaco y dura varios días, lo que dificulta la distinción de otras causas, y conlleva la administración empírica de anti- bióticos, la realización de pruebas diagnósticas innecesarias y aumenta el riesgo de repetidas administraciones del medicamento en el caso de no haberlo identificado ${ }^{4}$. El mecanismo implicado es desconocido aunque por sus características clínicas puede estar mediado por células $T$ activadas por el fármaco reactivo o sus metabolitos, unido de forma covalente o no covalente y presentado al complejo mayor de histocompatibilidad 5 .

El objetivo del presente trabajo es analizar la prevalencia y las características clínicas de la fiebre de origen inmunológico de un tipo peculiar de fármacos, los citostáticos, registrado en un Servicio de Oncología Médica durante un período de un año (julio 2001- julio 2002).

\section{Material y métodos}

Se analizaron las características clínicas de los pacientes con fiebre registradas en el Servicio de Oncología Médica de nuestro hospital, en los años 2001 y 2002.

La fiebre fue diagnosticada mediante el registro de la temperatura corporal.

Fue definida por la elevación mantenida de la temperatura corporal a $38^{\circ} \mathrm{C}$ o superior. Se realizó a cada paciente con fiebre un interrogatorio estandarizado por órganos que recogían los síntomas y signos acompañantes y se registraron cada una de las drogas que tomaba.

La revisión básica consistió en un hemograma, bioquímica (urea, creatinina, bilirrubina, GOT, GPT, FA, $\mathrm{LDH}, \mathrm{Na}, \mathrm{K}, \mathrm{Cl}, \mathrm{VSG}$, proteinograma y estudio de coagulación), radiografía de tórax, examen de esputo con 
tinción de hematoxilina/eosina, Ziehl-Neelsen y cultivo en medios apropiados en los casos con imágenes radiológicas compatibles con infección pulmonar $y / o$ síntomas respiratorios, análisis de orina elemental, urocultivo y hemocultivos.

De las 360 pacientes tratadas con quimioterapia entre 2001-2002 registramos 85 casos con fiebre que se ha distribuido en cinco grupos según su patogenia:

- Grupo A: 39 pacientes con fiebre neutropénica, diagnosticada por tener una cifra absoluta de neutrófilos inferior a $500 \mathrm{c} / \mathrm{mm}^{3}$.

- Grupo B: 30 pacientes con fiebre infecciosa no neutropénica, diagnosticados por criterios clínicos-radiológicos o confirmados por examen con tinción (esputo u orina) o cultivo.

- Grupo C: 5 pacientes tuvieron fiebre tumoral, definida por la ausencia de síntomas y signos que sugirieran un foco infeccioso, la demostración radiológica o analítica de progresión tumoral y la ineficacia al tratamiento antibiótico empírico.

- Grupo D: 6 pacientes tuvieron fiebre por fármacos de aparición precoz, diagnosticados por la presencia de fiebre entre los 30 minutos y las 24 horas siguientes a la introducción de la droga, con normalización espontánea o tras la toma de un antipirético en unas horas y reaparición al comenzar un nuevo ciclo de tratamiento. En estos casos se descartó la presencia clínica, analítica y radiológica de un foco infeccioso o progresión tumoral.

- Grupo E: 5 pacientes tuvieron fiebre por fármacos de aparición tardía, diagnosticados por la presencia de fiebre a las 48-72 horas siguientes de la introducción del fármaco y normalización espontánea e independiente de los antibióticos administrados. Además, se exigió que al menos en una ocasión previa hubiera tenido fiebre en las primeras 24 horas de la introducción del fármaco y se hubiera descartado la presencia clíni$\mathrm{ca}$, analítica y radiológica de un foco infeccioso o progresión tumoral.

\section{Resultados}

Se han estudiado un total de 85 pacientes con fiebre tras la administración de quimioterapia. La edad media fue de 57 años (rango 27-80) y 45 fueron varones (52\%). Todos habían sido diagnosticados de cáncer y recibían tratamiento de quimioterapia. Sesenta pacientes estaban en estadio diseminado y recibían el tratamiento con finalidad paliativa; el resto recibían quimioterapia adyuvante. Ningún paciente refería antecedentes alérgicos.
La prevalencia global de fiebre por fármacos ha sido del 12,9\% (11 casos), de los cuales 6 fue de aparición precoz y otros 5 tardía. La distribución por grupos ha sido como sigue: $39(45,8 \%)$ en el grupo $A$, $30(35 \%)$ en el grupo B, $5(5,8 \%)$ en el grupo C, 6 $(7 \%)$ en los grupo $D$ y $5(5,8 \%)$ en el grupo $E$.

En el grupo D, el tiempo medio de aparición de la fiebre fue de 5 horas desde el momento de la administración del tratamiento (rango 30 minutos-12 horas). Tres pacientes habían recibido oxaliplatino, uno etoposido y otros dos carboplatino. A todos se les había administrado previamente al citostático dexametasona y ondansetrón. Además de la fiebre, dos tuvieron disnea, dolor torácico opresivo y dolor lumbar. Tras la interrupción de la droga, cedieron los síntomas en unos 30 minutos (rango 30 minutos-1 hora). En la siguiente administración se repitieron los mismos síntomas en los seis pacientes. El tiempo medio de aparición de la clínica fue similar. En los cuatro pacientes con sólo fiebre se pudo administrar el citostático y recibieron paracetamol para la defervencencia. En los otros dos pacientes fue preciso la interrupción del tratamiento y hubo que suspenderlo posteriormente.

En el grupo $E$, el tiempo medio de aparición de la fiebre fue 48 horas (rango 48-72). La duración media fue de 3 días (rango 1-6), y ascendió por encima de $38^{\circ} \mathrm{C}$ (rango 38-39), tanto matutina como vespertina. El tratamiento citostático seguido fue el siguiente: FAC - 5-fluorouracilo, adriamicina, ciclofosfamida- 11 paciente), carboplatino-gemcitabina-navelbine- (1 pacientes), raltritexed (1 paciente), y oxaliplatino-5 fluorouracilo-leucovorin (2 pacientes). Todos habían recibido previamente a la administración del tratamiento de quimioterapia premedicación con ondansetrón y dexametasona (Tabla I).

La evolución fue independiente del tratamiento antibiótico administrado y respondió tras la administración de paracetamol o metamizol, aunque recidivó. Dos pacientes, a los tres días del comienzo de la fiebre, presentaron un exantema en la parte anterior de ambos muslos, abdomen, tórax y espalda, que se resolvió después de la defervescencia. Un paciente tuvo un exantema fijo en el brazo donde se administró el tratamiento (Tabla II).

Durante su estancia hospitalaria se descartó la presencia clínica, analítica y radiológica de un foco infeccioso o progresión tumoral.

Estos cinco pacientes previamente habían tenido fiebre de $38^{\circ} \mathrm{C}$ en las primeras 24 horas de la administración del fármaco y había cedido espontaneamente o con antipiréticos. En sucesivas administraciones siguieron tratamiento con prednisona y dexclorfeniramina no volviendo a presentar fiebre, aunque dos 
TABLA I

Características clínicas de los pacientes con fiebre de origen inmunológico retardado

\begin{tabular}{ccccccc}
\hline & Edad & Sexo & Antecedentes & Fármacos & Tumor & Estadio \\
\hline 1 & 66 & V & EPOC, Parkinson & Salbutamol, L-Dopa & Colon & II \\
2 & 55 & M & HTA & Atenolol & Mama & IIA \\
3 & 69 & M & & Colon & IV \\
4 & 63 & V & DMNID, Artritis & Glizipida, Deflacort & Pulmón & IIIB \\
5 & 50 & V & & & Colon & IV \\
\hline
\end{tabular}

\begin{tabular}{lccccc} 
Farmaco & Fiebre & Comienzo (horas) & Duración (días) & Evolución & Evolucion largo plazo \\
\hline Raltritexed & $\mathrm{SI}$ & 48 & 6 & Exantema & Dolor torácico \\
FAC & $\mathrm{SI}$ & 48 & 2 & & \\
$\begin{array}{l}\text { Oxaliplatino-5FU-LV } \\
\text { Carboplatino-vinorelbina- }\end{array}$ & $\mathrm{SI}$ & 48 & 2 & & \\
$\begin{array}{l}\text { gemcitabina } \\
\text { Oxaliplatino-5FU-LV }\end{array}$ & $\mathrm{SI}$ & 48 & 1 & Exantema & $\begin{array}{c}\text { Manchas acrómicas en dedos } \\
\text { SI }\end{array}$ \\
\hline
\end{tabular}

TABLA II

\begin{tabular}{lc}
\hline \multicolumn{2}{c}{ Características de los episodios de fiebre } \\
\hline & Pacientes \\
\hline Fiebre & $5 / 5$ \\
Exantema & $3 / 5$ \\
Leucocitosis & $5 / 5$ \\
Eosinofilia & $1 / 5$ \\
Alteración renal & $0 / 5$ \\
Alteración hepática & $0 / 5$ \\
Eficacia corticoides & $5 / 5$ \\
Exitus & $0 / 5$ \\
\hline
\end{tabular}

pacientes presentaron con posterioridad máculas acrómicas de 2 a $4 \mathrm{~cm}$ de diámetro, localizadas en dedos de las manos y dorso de brazos y otro paciente tuvo un dolor torácico opresivo sin cambios electrocardiográficos ni elevación enzimatica (CPK, CPK-MB, Troponina T) a los cuatro días de la administración del tratamiento.

\section{Discusión}

La fiebre ha sido una de las causas más frecuentes de ingreso hospitalario en oncología desde el inicio de la utilización de la quimioterapia citotóxicab ${ }^{6}$, supo- niendo unos costes considerables por las pruebas diagnósticas y el tratamiento empírico realizado.

En la mayoría de las ocasiones la infección es la causa de fiebre, en relación con el descenso de la cifra de neutrófilos y el daño de la integridad de la mucosa gastrointestinal por la quimioterapia. Sin embargo, en los últimos años la fiebre secundaria a fármacos se ha vuelto cada vez más frecuente, en relación con la actividad farmacológica del medicamento o mediado por un mecanismo inmunológico específico ${ }^{7}$.

Determinados medicamentos (antibióticos $\beta$-lactámicos) inducen la formación de anticuerpos lgE específcos que se unirán a la supercífie de los mastocitos y basófilos, induciendo su degranulación tras sucesivas administraciones ${ }^{8}$. En otras ocasiones, los fármacos (por ejemplo, antiinflamatorios no esteroideos), producen alteración del metabolismo del ácido araquidónico liberando potentes mediadores (prostaglandinas y leucotrienos). Existe también la posibilidad de que el fármaco (por ejemplo, opiáceos y anestésicos) induzca directamente la degranulación del mastocito y la subsiguiente liberación de mediadores.

Sin embargo, cada día existe más evidencia de la participación de mecanismos celulares mediados por linfocitos $T$ activados. Estudios recientes muestran que los individuos presentan linfocitos $T$ en sangre periférica con aumento de la expresión de marcadores de activación como HLA-DR, CD25 y CD699. Asimismo, se ha observado que en pacientes con lesiones cutáneas 
de hipersensibilidad retardada, existe una subpoblación de linfocitos $T$ circulantes que expresa en su superficie el antígeno CLA que interacciona con la E-selectina endotelial de las lesiones cutáneas. La exposición repetida al alergeno condicionará una sobreexpresión en los linfocitos $T$ de la molécula CLA, focalizando la recirculación de estos en la piel ${ }^{10}$.

Aunque la mayoría de los fármacos son capaces de producir reacciones alérgicas, los antibióticos $\beta$-lactámicos y los antiinflamatorios no esteroideos constituyen la causa más frecuente de reacciones adversas a fármacos mediados por un mecanismo inmunológico específico"1.

La hipersensibilidad por citostáticos ha sido poco estudiada, a pesar de que el número de reacciones alérgicas no es despreciable ${ }^{12}$. Sin embargo, las manifestaciones clínicas no son exclusivas de las reacciones inmunitarias inducidas por fármacos y son frecuentemente atribuidas a síntomas y signos relacionados con la enfermedad subyacente, a reacciones idiosincrásicas o fenómenos tóxicos infrecuentes del fármaco. Además, suele ser difícil asegurar que una reacción clínica es debida a hipersensibilidad al citostático, al no disponer de pruebas estandarizadas (IgE-RAST, pruebas cutáneas y/o pruebas de provocación) o ser secundaria a metabolitos intermedios formados en el hígado o en las células fagocitarias, lo que dificulta la reproducción in vitro. Por ello, es fundamental para el diagnóstico una anamnesis cuidadosa que especifique la secuencia de eventos desde el comienzo de la terapia a la aparición de los síntomas. Será difícil tipificar una determinada reacción de hipersensibilidad según la clasificación de Gell-Coombs ${ }^{13}$ ya que pueden estar mediadas por más de un mecanismo. Sin embargo, hay muchos casos de reacciones a drogas que se adecúan a esta clasificación siendo útil para el diagnóstico la presencia de manifestaciones clínicas específicas. Así, la aparición precoz o en menos de 24 horas hará pensar en un mecanismo tipo I, mediado por lgE y la aparición tardía después de 48 horas de la administración del fármaco, en un mecanismo de hipersensibilidad celular o tipo IV.

En nuestra serie se identificaron once casos de fiebre por fármacos de origen inmunológico, de los cuales los seis incluidos en el grupo $D$, pudieron ser atribuidos a un mecanismo inmunológico lgE al presentarse precozmente tras su administración, y los cinco pacientes en el grupo $E$, por un mecanismo celular dado el momento de aparición de la fiebre y la presencia de signos cutáneos compatibles.

La evolución clínica de los enfermos del primer grupo fue la esperada por la aparición de síntomas similares a pesar de la premedicación con corticoides,que obligó a la suspensión del tratamiento en dos pacientes con síntomas clínicos sugerentes de anafilaxia.

Asimismo, los enfermos del grupo $E$, con fiebre prolongada ingresaron por sospecha de infección y recibieron tratamiento antibiótico. La aparición posterior de manifestaciones cutáneas (exantema generalizado o exantema fijo) en tres pacientes, la ineficacia del tratamiento antibiótico y el control con prednisona sugirió un posible origen inmunológico.

El papel de la hipersensibilidad a fármacos ha sido puesta de relieve en varios trabajos ${ }^{14,15}$. Sin embargo, en la práctica médica apenas es considerada, en cierto modo por la ausencia de pruebas diagnósticas y por la necesidad de la administración de dichos medicamentos. Todo esto hace que siga siendo la historia clínica el pilar fundamental sobre el que se basa el diagnóstico de alergia a fármacos y en muchos casos la única herramienta de que disponemos.

En la literatura internacional las reacciones a fármacos se especifican cuando supone un evento adverso grave que conlleva la retirada del fármaco, por lo que la incidencia real está menospreciada. Así, Pazdur et $\mathrm{al}^{16}$ en un estudio fase II con raltritexed en 42 pacientes con cáncer de páncreas avanzado, contabiliza 4 reacciones alérgicas graves aunque no describen las manifestaciones clínicas. En el estudio $\mathrm{MOSAIC}^{17}$ con oxaliplatino/5FU/LV adyuvante en pacientes con cáncer de colon se observaron dos reacciones alérgicas grado 3-4, lo que supuso un $2 \%$, porcentaje inferior al nuestro, posiblemente por considerar sólo las reacciones graves.

Parece pues, que las reacciones por hipersensibilidad no se reflejan adecuadamente en la bibliografía, y mucho menos se describen las manifestaciones clínicas, a pesar de que su estudio ayudaría a identificarlas evitando la administración de medicamentos inadecuados y la realización de pruebas diagnósticas innecesarias, además de conocer la importancia del mecanismo implicado en la alergia a fármacos.
Correspondencia:
Dr. M. J. Oruezábal Moreno
Sección Oncología Médica
Hospital La Mancha Centro
C/ Constitución, s/n
E-13600 Alcázar de San Juan (Ciudad Real)
E-mail: mrburon@hotmail.com 


\section{Bibliografía}

1. Bernstein JA. Allergic drug reactions. Postgrad Med 1995; 98 (2):159-166.

2. Rebecca G. Advances in allergic diseases: An update for the new millenium. Understanding drug allergies. Journal of Allergy and Clinical Immunology. 2000; 105 (6).

3. Lipsky BA, Hirschmann JV. Drug fever. JAMA 1981; 245: 851-854.

4. Atkins E, Francis L. Pathogenesis of fever in delayed hypersensitivity:factors influencing release of pyrogen-inducing lymphokines. Infect Immun 1978; 21:808-812.

5. Larry B, Stephen A. Drug hypersensitivity. Immune mechanisms of drug allergy. Immunology and Allergy Clinics of North America. 1998; 18 (4).

6. Carrato A, Barón JM, Díaz N. Complicaciones metabólicas en el enfermo con cáncer I: fiebre de origen tumoral. En: González Barón, Ordóñez, Feliú, Zamora, Espinosa, eds. Tratado de medicina paliativa. Madrid: Panamericana, 1996; 529-538.

7. Tabor PA. Drug induced fever. Drug Intell Clin Pharm 1986; 20:413-420.

8. Caldwell JR, Cluff LE. Adverse reactions to antimicrobial agents. JAMA 1974; 230:77-80.

9. Sanders ME, Makgoba MW, Sharrow SO, Stephany D, Springer TA, Young HA et al. Human memory T-lymphocytes express increased levels of three cell adhesion molecules (LFA-3, CD2, and LFA-1) and three other molecules (UCHLI, CDw29, and Pgp-1) and have enhanced IFN- $\gamma$ production. J Immunol 1988; 140:1401-1407.
10. Kehren J, Desvignes C. Cytotoxicity is mandatory for CD8 T cell mediated contact hypersensitivity. J Exp Med 1999; 189:779-786.

11. Miller RR, Greenblatt DJ, editores. Drug effects in hospitalizad patients. Experiences of the Boston Collaborative Drug Surveillance Program 1966-1975. Nueva York: Wiley and Sons, 1976.

12. Sato A, Sakashita A, Taguchi S. Hypersensitivity reactions to cancer chemotherapeutic agents. Gan To Kagaku Ryoho. 2003;30(6):793-800.

13. Griffin JP. Drugs induced allergic and hipersensibility reactions. En latrogenic Diseases. Oxford Medical Publications, Oxford, 1986.

14. Ulrich-Pur H, Fiebiger WCC, Schüll B. Oxaliplatin induced fever and release of IL-6. Oncology 2000; 59:187189.

15. Thomas RR, Quinn MG, Schuler B. Hypersensitivity and idiosyncratic reactions to oxaliplatin. Cancer 20031 ; 97(9):2301-2307.

16. Pazdur R, Meropol NJ, Casper ES, Fuchs C, Douglass $\mathrm{HO}$, Vincent $M$, et al. Phase II trial of ZD 1694in patients with advanced pancreatic cancer.Investigational New Drugs 1995; 13(4):355-358.

17. Gramont A, Boni C, Navarro M, Tabernero J, Hickish T, Topham C, et al. Oxaliplatin/5-fu/lv in adjuvant colon cancer: safety results of the international randomized Mosaic trial. Proceedings of American Society of Clinical Oncology; 2002, May; 1015. 